\title{
FINITELY CYCLIC HOMOGENEOUS CONTINUA
}

\author{
PAWEL KRUPSKI AND JAMES T. ROGERS, JR.
}

(Communicated by Dennis Burke)

\begin{abstract}
A curve is finitely cyclic if and only if it is the inverse limit of graphs of genus $\leq k$, where $k$ is some integer. In this paper it is shown that if $X$ is a homogeneous finitely cyclic curve that is not tree-like, then $X$ is a solenoid or $X$ admits a decomposition into mutually homeomorphic, homogeneous, tree-like continua with quotient space a solenoid. Since the Menger curve is homogeneous, the restriction to finitely cyclic curves is essential.
\end{abstract}

A continuum is a compact, connected, nonvoid metric space. A curve is a one-dimensional continuum. Each curve is an inverse limit of finite graphs.

A curve is said to be arc-like if it is an inverse limit of arcs, circle-like if it is an inverse limit of circles, and tree-like if it is an inverse limit of trees. We assume that the bonding maps of an inverse sequence are surjective.

A curve $X$ is homogeneous if for each pair of points $x$ and $y$ of $X$, there exists a homeomorphism of $(X, x)$ onto $(X, y)$. A solenoid is an inverse limit of circles with all the bonding maps being covering maps. Under this definition, the circle is a solenoid. Each solenoid is homogeneous, since it is a topological group.

A curve is called simply cyclic if it is an inverse limit of graphs each of which contains only one cycle. Hence simply cyclic curves include circle-like curves as a proper subset. The second author [7] has shown that if a simple cyclic, homogeneous curve is not tree-like, then either it is a solenoid or it admits a decomposition into mutually homeomorphic, tree-like, homogeneous curves with quotient space a solenoid.

A curve is called finitely cyclic if it is an inverse limit of graphs of genus less than $k$, where $k$ is some natural number. The purpose of this note is to prove the following theorem:

Theorem. If a finitely cyclic, homogeneous curve is not tree-like, then either it is a solenoid or it admits a decomposition into mutually homeomorphic, tree-like, homogeneous curves with quotient space a solenoid.

Received by the editors April 24, 1990.

1980 Mathematics Subject Classification (1985 Revision). Primary 54F20; Secondary 54F50.

Key words and phrases. Continuum, curve, homogeneous, terminal subcontinuum, decomposition, simply cyclic, finitely cyclic.

The second author was partially supported by NSF Grant DMS-8600364. 
Since R. D. Anderson [1] has shown that the Menger curve is homogeneous, it follows that the restriction to finitely cyclic curves cannot be dropped, and so the result is the best possible in this sense. The ideas of the proof are completely different from those in [7].

In the proofs, we prefer to deal with open covers of curves as opposed to inverse limits. An equivalent definition for a curve to be tree-like, for instance, is that it admits finite open covers of arbitrarily small mesh whose nerves are trees. Other definitions and background for this problem may be found in [ 5 , $8]$. Related work may be found in $[2-4,6]$.

A continuum $X$ is said to be somewhat tree-like if there is a number $\varepsilon>$ 0 and a sequence of open covers $\left\{\mathscr{C}_{n}\right\}$ of $X$ such that $\mathscr{C}_{n+1}$ refines $\mathscr{C}_{n}$, mesh $\mathscr{C}_{n} \rightarrow 0$ and each cover $\mathscr{C}_{n}$ contains a tree-like chain $\mathscr{T}_{n}$ such that $\operatorname{diam} \bigcup \mathscr{T}_{n}>\varepsilon$ and $\bigcup\left(\mathscr{C}_{n} \backslash \mathscr{T}_{n}\right) \cap \bigcup \mathscr{T}_{n}$ is contained in only one end-link of $\mathscr{T}_{n}$.

All nondegenerate tree-like continua as well as simply cyclic noncircle-like continua are somewhat tree-like.

Theorem 1. If a somewhat tree-like continuum $X$ is homogeneous, then $X$ contains a sequence of nondegenerate subcontinua $Y_{k}$ such that for each $k$ :

(i) $Y_{k}$ has an outlet point;

(ii) $Y_{k+1} \subset Y_{k}$;

(iii) $\bigcap_{k=1}^{\infty} Y_{k}$ is degenerate.

In particular $X \in \mathscr{F}$, which means that $X$ contains a sequence of nondegenerate subcontinua $Y_{k}$ converging to a point such that each $Y_{k}$ has an outlet point and $\bigcap_{k=1}^{\infty} Y_{k} \neq \varnothing$.

Proof. See the proof of [5, Theorem 3.4].

We say that small subcontinua of the continuum $X$ have a topological property $P$ if there exists a positive number $\varepsilon$ such that any subcontinuum of $X$ of diameter less than $\varepsilon$ has the property $P$.

Theorem 2. If $X$ is a nondegenerate, homogeneous continuum such that small subcontinua of $X$ are unicoherent, then $X$ contains either an arc or arbitrarily small, nondegenerate, indecomposable subcontinua.

Proof. Apply the proof of [5, Theorem 5.2].

Theorem 3. If $X \in \mathscr{F}$ is homogeneous and small subcontinua of $X$ are unicoherent, then $X$ contains arbitrarily small, nondegenerate terminal subcontinua.

Proof. Apply the proof of [5, Theorem 5.12].

A sequence $\left\{\mathscr{C}_{n}\right\}$ of finite open covers of a curve $Y$ is said to satisfy condition $(\mathscr{D})$, denoted $\left\{\mathscr{C}_{n}\right\} \in(\mathscr{D})$, if

(1) $\mathscr{C}_{n+1}$ refines $\mathscr{C}_{n}$;

(2) $\operatorname{mesh} \mathscr{C}_{n} \rightarrow 0$; and 
(3) there is an integer $N$ such that, for each $n$, the nerve of $\mathscr{C}_{n}$ is a graph of genus $\leq N$.

Define $e(Y)=\min \left\{N\right.$ : there exists $\left\{\mathscr{C}_{n}\right\} \in(\mathscr{D})$ with genus $\mathscr{C}_{n} \leq N$, for each $n\}$. A sequence $\left\{\mathscr{C}_{n}\right\}$ realizing this minimum bound is called essential.

It follows that $e(Y)=0$ if and only if $Y$ is tree-like, and $e(Y)=1$ if and only if $Y$ is simply cyclic and not tree-like.

A curve $Y$ is finitely cyclic if some sequence of finite covers of $Y$ satisfies condition $(\mathscr{D})$.

Theorem 4. If $Y$ is a finitely cyclic curve, then small subcontinua of $Y$ are tree-like.

Proof. Consider an essential sequence of covers $\left\{\mathscr{C}_{n}\right\}$ of $Y$. If $e(Y)=0$, then all subcontinua of $Y$ are tree-like, so we may assume $e(Y)>0$.

We claim there exists $\varepsilon>0$ such that for each $n$, every circular chain $\mathscr{D}_{n} \subset \mathscr{C}_{n}$ satisfies diam $\bigcup \mathscr{D}_{n}>\varepsilon$. Indeed, suppose there is a sequence of circular chains $\left\{\mathscr{D}_{n}\right\}$ such that $\mathscr{D}_{n} \subset \mathscr{C}_{n}$ and $\operatorname{diam} \bigcup \mathscr{D}_{n} \rightarrow 0$ as $n \rightarrow \infty$. Define new covers $\mathscr{C}_{n}^{\prime}$ of $Y$ by

$$
\mathscr{C}_{n}^{\prime}=\left\{\bigcup \mathscr{D}_{n}\right\} \cup\left(\mathscr{C}_{n}-\mathscr{D}_{n}\right) \text {. }
$$

For each cover $\mathscr{C}_{n}^{\prime}$, genus $\mathscr{C}_{n}^{\prime}=$ genus $\mathscr{C}_{n}-1$. A suitable subsequence of $\left\{\mathscr{C}_{n}^{\prime}\right\}$ satisfies condition $(\mathscr{D})$; this contradicts the definition of $e(Y)$. Hence such an $\varepsilon$ exists.

If $Z$ is a subcontinuum of $Y$ and $\operatorname{diam} Z<\varepsilon$, then, for large $n$,

$$
\mathscr{C}_{n}(Z)=\left\{C \in \mathscr{C}_{n}: C \cap Z \neq \varnothing\right\}
$$

is an open cover of $Z$ that contains no circular chain. Therefore, each $\mathscr{C}_{n}(Z)$ is a tree chain, and $Z$ is tree-like.

Theorem 5. If a nondegenerate finitely cyclic continuum $Y$ is not somewhat treelike, then there is an essential sequence $\left\{\mathscr{C}_{n}\right\}$ such that nerve $\mathscr{C}_{n}$ contains no endpoints, for each $n$. Moreover, there exists a natural number $k$ such that for each $n$ the number of ramification points of nerve $\mathscr{C}_{n}$ is less than $k$.

Theorem 6. If a homogeneous finitely cyclic continuum $Y$ is not somewhat treelike, then small subcontinua of $Y$ are arc-like.

Proof. Suppose there exists a sequence of tree-like continua $T_{n} \subset Y$ such that diam $T_{n} \rightarrow 0$ and no continuum $T_{n}$ is arc-like (see Theorem 4 ). By the homogeneity of $Y$, we can assume that for every point $x \in Y$ there is a sequence of tree-like subcontinua $T_{n}(x)$ which are not arc-like and $\lim _{n} T_{n}(x)=\{x\}$. Therefore we can find a sequence of tree-like subcontinua $T_{n}$ which are not arc-like and are mutually disjoint. Consider an essential sequence of covers $\left\{\mathscr{C}_{n}\right\}$ satisfying the conclusion of Theorem 5 . There exists $m$ such that for all $n>m$ and for all $i=1, \ldots, k$, the continuum $T_{i}$ is covered by a tree chain $\mathscr{T}_{n}^{i} \subset \mathscr{C}_{n}$ that is not a chain and $\mathscr{T}_{n}^{i} \cap \mathscr{T}_{n}^{j}=\varnothing$, if $i \neq j$. To each 
$i=1, \ldots, k$, assign a ramification point of nerve $\mathscr{T}_{n}^{i}$. This correspondence is one-to-one; this contradicts Theorem 5.

Theorem 7. If a homogeneous finitely cyclic nondegenerate continuum $Y$ contains no terminal, proper, nondegenerate subcontinua, then small subcontinua of $Y$ are arc-like.

Proof. This is a consequence of Theorem 6, since $Y$ is not somewhat tree-like by Theorems 1,3 , and 4 .

The next two theorems are proved in [2].

Theorem 8. If $X$ is a nondegenerate homogeneous continuum with no proper nondegenerate terminal subcontinua, then the following conditions are equivalent:

(a) small subcontinua of $X$ are atriodic and unicoherent;

(b) small subcontinua of $X$ are decomposable and arc-like;

(c) small subcontinua of $X$ are arc-like.

Theorem 9. If a homogeneous continuum $X$ contains an arc, then the following conditions are equivalent:

(a) small subcontinua of $X$ are atriodic;

(b) small subcontinua of $X$ are arcs;

(c) $X$ is a solenoid.

Theorem 10. If a homogeneous finitely cyclic continuum $Y$ contains no terminal, proper, nondegenerate subcontinuum, then $Y$ is a solenoid.

Proof. It follows from Theorems 7 and 8 that small subcontinua of $Y$ are decomposable, atriodic, and unicoherent. By Theorem 2, the continuum $Y$ contains an arc, and Theorem 9 implies $Y$ is a solenoid.

Theorem 11. If $X$ is a homogeneous finitely cyclic continuum that is not tree-like, then $X$ is a solenoid or $X$ admits a decomposition into mutually homeomorphic, homogeneous tree-like continua with quotient space a solenoid.

Proof. If $X$ contains no terminal, proper, nondegenerate subcontinuum, then $X$ is a solenoid, by Theorem 10 . Otherwise the Terminal Decomposition Theorem ([5] or [9]) implies that $X$ admits a decomposition into mutually homeomorphic, homogeneous tree-like continua with quotient space a homogeneous curve $Y$ that contains no terminal, proper, nondegenerate subcontinuum. It follows from a result of $\mathrm{J}$. W. Rogers, Jr. [10, Theorem 3] that $Y$ is finitely cyclic. Hence $Y$ is a solenoid.

\section{REFERENCES}

1. R. D. Anderson, A characterization of the universal curve and a proof of its homogeneity, Ann. of Math. (2) 67 (1958), 313-324.

2. E. Duda, P. Krupski, and J. T. Rogers, Jr., On locally chainable homogeneous continua, Topology Appl. (to appear). 
3. C. L. Hagopian, Atriodic homogeneous continua, Pacific J. Math. 113 (1984), 333-347.

4. C. L. Hagopian and J. T. Rogers, Jr., A classification of homogeneous circle-like continua, Houston J. Math. 3 (1977), 471-474.

5. P. Krupski and J. R. Prajs, Outlet points and homogeneous continua, Trans. Amer. Math. Soc. 318 (1990), 123-141.

6. W. Lewis, Homogeneous tree-like continua, Proc. Amer. Math. Soc. 82 (1981), 470-472.

7. J. T. Rogers, Jr., Simply cyclic homogeneous non-tree-like curves decompose to solenoids, Proc. Amer. Math. Soc. 108 (1990), 1059-1062.

8. _ Homogeneous continua, Topology Proc. 8 (1983), 213-223.

9. __ Decomposition of continua over the hyperbolic plane, Trans. Amer. Math. Soc. 308 (1988), 277-291.

10. J. W. Rogers, Jr., Monotone maps and e-maps, Topology Proc. 4 (1979), 533-540.

Department of Mathematics, University of Miami, Coral Gables, Florida 33124

Current address: Mathematical Institute, University of Wroclaw, Pl. Grunwaldzki 2/4, 50-384 Wrocław, Poland

Department of Mathematics, Tulane University, New Orleans, Louisiana 70118 